\title{
Occlusion of canine atrial appendage using an expandable silicone band
}

\author{
Patrick M. McCarthy, MD, FACC, ${ }^{a}$ Richard Lee, MD, MBA, ${ }^{a}$ Jessica L. Foley, PhD, ${ }^{\mathrm{b}}$ \\ Lynette Phillips, DVM, PhD, ${ }^{\mathrm{b}}$ Tessy Kanayinkal, MS, ${ }^{\mathrm{b}}$ and David E. Francischelli, MS ${ }^{\mathrm{b}}$
}

Objective: Because the left atrial appendage is thought to be a major source of stroke in patients with atrial fibrillation, a new device and technique were developed for thoracoscopic isolation of the left atrial appendage.

Methods: The left and right atrial appendages were approached from an 11.5-mm port in the left thorax in 15 canines. With an atraumatic grasper for appendage positioning, expandable silicone bands covered with polyester fabric were placed at the base of the left and right atrial appendages. The location of the bands was marked with radiopaque clips to assess migration, and radiopaque dye was injected to confirm occlusion. The animals were killed at 1 week $(n=3), 2$ weeks $(n=6)$, or 12 weeks $(n=6)$.

Results: The bands were deployed on 30 appendages without complications. The appendages were $100 \%$ occluded, and there was no migration of any bands at death. There was no indication of bleeding, rupture, or systemic emboli in any of the 15 animals. Following complete occlusion, the appendages became necrotic and were replaced by scar tissue. The healed atrial surface was consistently smooth and devoid of thrombus. There was evidence of mild-to-moderate inflammation associated with a foreign body-type reaction to the fabric material but no pericarditis.

Conclusions: Permanent occlusion of the left atrial appendage is feasible via a thoracoscopic epicardial approach with this novel silicone band. As the atrial tissue becomes necrotic, the silicone band continues to constrict, ensuring that the appendage remains isolated. Band occlusion of the left atrial appendage could potentially improve overall treatment outcome in patients at high risk of atrial thrombus formation. (J Thorac Cardiovasc Surg 2010;140:885-9)

The left atrial appendage (LAA) is a common site of thrombus formation in patients with cardiovascular disease. ${ }^{1,2}$ Surgical exclusion of the LAA is recommended in the current American Heart Association/American College of Cardiology guidelines for patients with mitral valve disease at the time of mitral valve surgery. ${ }^{3}$ Recently, the Left Atrial Appendage Occlusion Study demonstrated that the use of sutures or a stapling device to occlude the LAA at the time of coronary artery bypass grafting could be performed without increasing the duration of cardiopulmonary bypass or the risk of postoperative complications. ${ }^{4}$ Although surgical techniques to excise or isolate the LAA are easy to apply, the reproducibility and efficacy of the techniques available have not been clearly established. Katz and colleagues ${ }^{5}$ reported

\footnotetext{
From the Bluhm Cardiovascular Institute, ${ }^{\text {a }}$ Division of Cardiothoracic Surgery at Northwestern University Feinberg School of Medicine and Northwestern Memorial

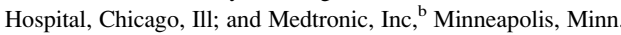

Supported by Medtronic, Inc, Minneapolis, Minn.

Disclosures: Dr Richard Lee is a consultant for Medtronic, Inc. Jessica Foley, Lynette

Phillips, Tessy Kanayinkal, and David Francischelli are employees of Medtronic, Inc. Received for publication Sept 4, 2009; revisions received Dec 22, 2009; accepted for publication Jan 2, 2010; available ahead of print March 26, 2010.

Address for reprints: Patrick M. McCarthy, MD, FACC, Division of Cardiothoracic Surgery, Northwestern University, 201 East Huron St, Suite 11-140 Chicago, IL 60611-2908 (E-mail: pmccart@nmh.org).

0022-5223/\$36.00

Copyright (c) 2010 by The American Association for Thoracic Surgery doi:10.1016/j.jtcvs.2010.01.015
}

that surgical ligation of the LAA with a running suture in 50 patients undergoing mitral valve surgery was only effective in excluding the LAA in $64 \%$ of the patients. Furthermore, García-Fernández and colleagues ${ }^{6}$ ligated the LAA by using a double-suture technique at the time of mitral valve replacement surgery in 58 patients and found that ligation was incomplete in 6 patients. A more recent study by Kanderian and colleagues ${ }^{7}$ compared the success rate of surgical excision of the LAA with suture or stapler exclusion in $137 \mathrm{pa}-$ tients undergoing open-heart surgery. They reported that successful closure occurred more often with excision $(73 \%)$ than with suture exclusion $(23 \%)$ or stapler exclusion $(0 \%)$.

In addition to surgical exclusion of the LAA via an openchest approach, a thoracoscopic approach has been used to obliterate the LAA. ${ }^{8} 9$ Thoracoscopic LAA exclusion has been performed with both a loop snare and a stapler in a small number of patients. ${ }^{9}$ A thoracoscopic epicardial approach has the potential advantages of simplicity and a low procedural risk of embolism; however, there is a need for a device to facilitate rapid, reliable, and safe epicardial occlusion of the LAA. Therefore the goal of the present study was to develop a new epicardial band occlusion device and investigate its utility to occlude canine atrial appendages. An additional objective was to investigate the long-term (up to 12 weeks) healing response of the atria and appendages after band occlusion. 


\section{Abbreviations and Acronyms \\ LAA $=$ left atrial appendage \\ RAA $=$ right atrial appendage}

\section{MATERIALS AND METHODS Delivery Device and Silicone Band}

A thoracoscopic device made of acrylonitrile butadiene styrene, stainless steel, and polytetrafluoroethylene was used to deliver an expandable silicone band $(0.225 \mathrm{inch})$ around the base of the atrial appendage (Figure 1). The band was attached to the distal end of the delivery device by a Nitinol wire. After the deployment of the band, the Nitinol wire and delivery device were removed from the surgical site. The occlusion bands were rectangular in cross-sectional shape, white in color, and made of silicone embedded with $\mathrm{BaSO}_{4}$. The bands were covered with polyester fabric to reduce the possibility of migration after implantation. The "hole" in the middle of the band is $5.7 \mathrm{~mm}$ when released.

\section{Implant Procedure}

Silicone bands were implanted in 15 adult mongrel dogs of either sex weighing 25 to $34 \mathrm{~kg}$. All animals have received humane care in compliance with the guide for the care and use of laboratory animals and the protocol was approved by the Institutional Animal Care and Use Committee. All procedures and animal housing were at Medtronic's Physiological Research Laboratories. An analgesic and sedative (morphine and acepromazine) was given to each animal before the induction of anesthesia. The animals were induced with propofol, intubated, and then ventilated with isoflurane $(1.5 \%$ to $3 \%)$ to maintain a surgical plane of anesthesia. The left atrial appendate (LAA) and right atrial appendage (RAA) were approached from an 11.5 -mm port in the left fourth intercostal space. Baseline images of the RAA and LAA and the surgical procedure were recorded by means of thoracoscopic video. An atraumatic grasper was used to position the appendage so an expandable occlusion band $(0.225$ inch ID) could be placed at the base of each appendage. The occlusion band was positioned and deployed on both atrial appendages using a thoracoscopic delivery device (Figure 1). The occlusion was assessed visually by ascertaining that the band caused isolation of the appendage. There were no instances of hemodynamic instability. Hemoclips were placed on the proximal side of the band after implantation to mark the position of the band. A pigtail catheter was introduced into the right atrium through jugular access for the administration of radiopaque dye. Fluoroscopic images of the heart were obtained during dye injection before and after implantation to determine the completeness of occlusion. Animals recovered under the supervision of a veterinarian and were given aspirin ( $81 \mathrm{mg}$ administered orally) and dipyridamole (50 mg administered orally) daily for 1 week after implantation. Animals receiving implants were killed after 1 week $(n=3), 2$ weeks $(n=6)$, or 12 weeks $(n=6)$. Additional fluoroscopic images were obtained just before death.

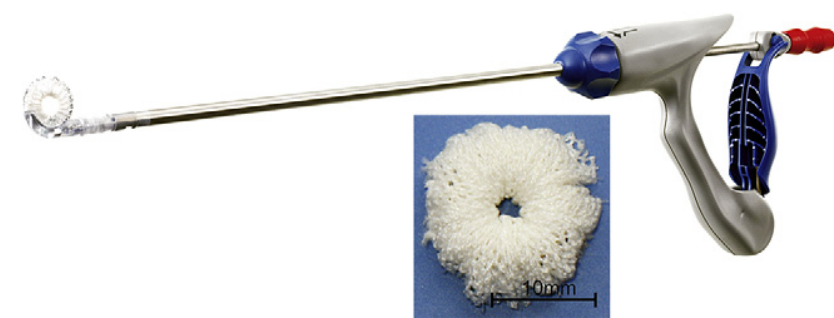

FIGURE 1. Photographs of the delivery device and occlusive band. The delivery device is shown with the preloaded, polyester fabric-covered silicone band. The inset shows a close-up view of the occlusion band after release from the delivery device.

\section{Pathology and Histology}

Following termination, the kidneys, liver, lungs, and brain from each animal were grossly examined, and representative samples of each organ were fixed in $10 \%$ neutral buffered formalin. The endocardial and epicardial aspects of each heart were grossly examined. If the band was not located at the base of the appendage, the distance to the previously placed hemoclips was recorded. The left and right atria, with the bands in situ, were dissected away from the heart and fixed in $10 \%$ neutral buffered formalin. After fixation, each appendage remnant was bisected to assess the cross-sectional appearance of the tissues. The appendage tissues were serially sampled for evaluation of the occluded appendages, tissues through the constricted zone, and the appendage/atrial tissue proximal to the constricted zone. All fixed tissue samples were routinely processed, paraffin embedded, sectioned via microtome at $4 \mu \mathrm{m}$ thickness, and mounted on glass slides for light microscopy examination. Appendage sections were stained with hematoxylin and eosin and Masson's trichrome and evaluated for fibrosis, inflammation, remaining cardiac tissue, and the presence of any thrombotic material on the endocardial surface, with documentation of any additional pathology. Sections of other organs were stained with hematoxylin and eosin and evaluated for vascular thromboembolism, with documentation of any additional pathology.

\section{RESULTS}

Bands were placed without complication on all the appendages; 2 bands were placed on 1 appendage each (1 RAA and 1 LAA) in 2 animals. The extra band on the RAA of one animal was placed intentionally to determine the feasibility of placing a second band. A second band on the LAA in one animal was necessary because the first band was not placed all the way at the base of the appendage. Fluoroscopic images obtained during the injection of radiopaque dye before and after band implantation showed that occlusion was $100 \%$ effective in both the RAA and
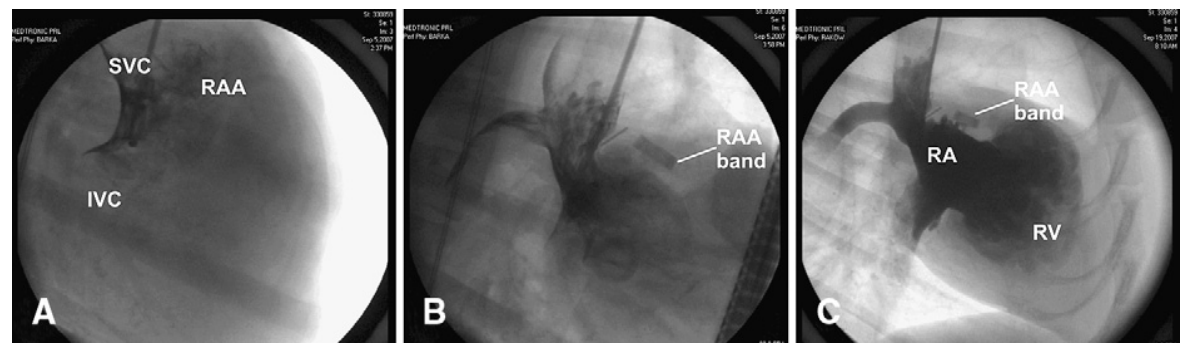

FIGURE 2. Fluoroscopic images of right atrium with dye injection before (A), immediately after (B), and 2 weeks after (C) band implantation. SVC, Superior vena cava; $I V C$, inferior vena cava; $R A A$, right atrial appendage; $R A$, right atrium; $R V$, right ventricle. 

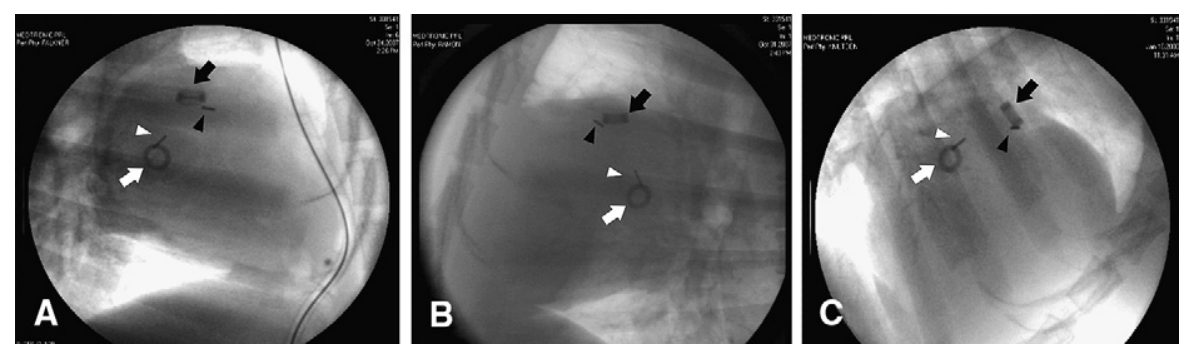

FIGURE 3. Fluoroscopic images of the heart at implantation (A) 1 week (B) and 12 weeks (C) after implantation. The left atrial bands are indicated by white arrows, and the right atrial bands are indicated by black arrows. Hemoclips placed on the left and right atria are indicated by white and black triangles, respectively.

\section{left appendage}
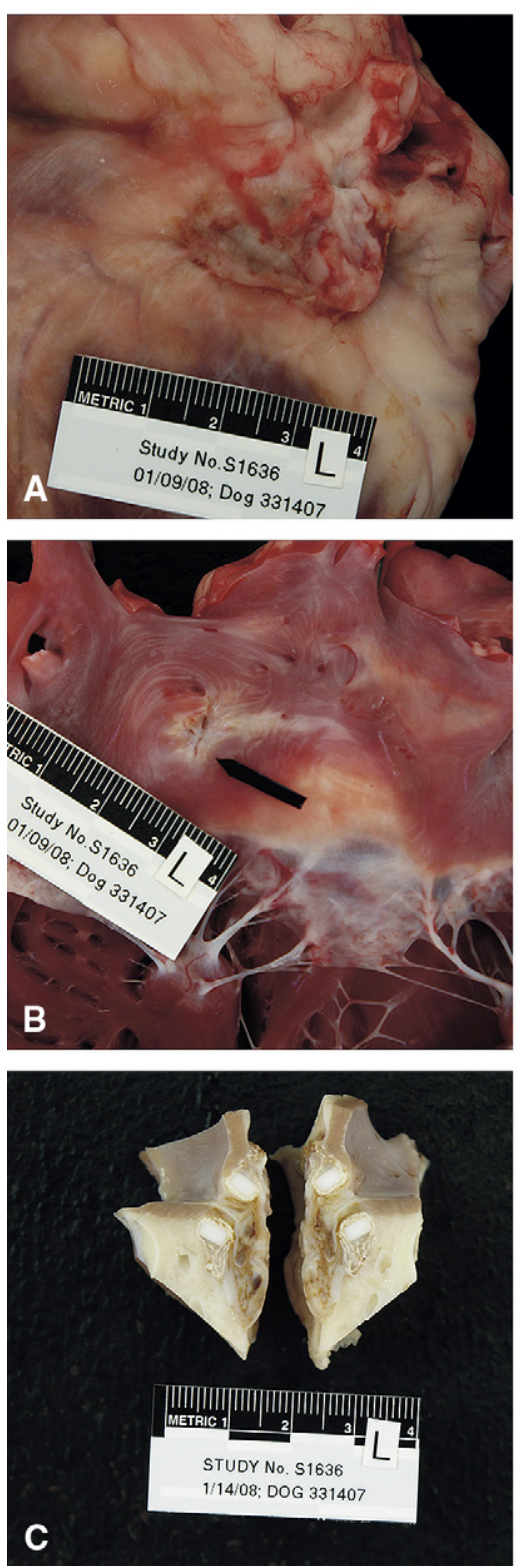

right appendage
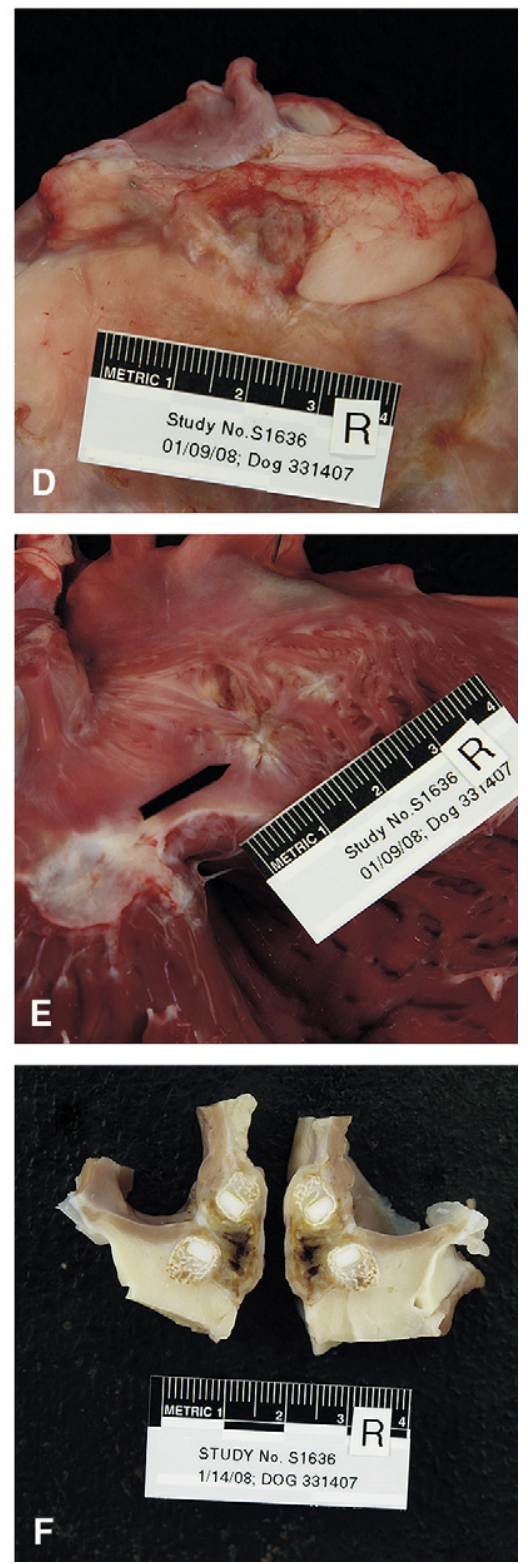

FIGURE 4. Gross pathology 12 weeks after implantation of polyester fabric-covered bands. A, Left atrial appendage overview. B, Opened left atrium. The arrow points to the endocardial occlusion site. C, Bisected left atrial appendage and occluding band. D, Right atrial appendage overview. E, Opened right atrium. F, Bisected right atrial appendage and occluding band. 

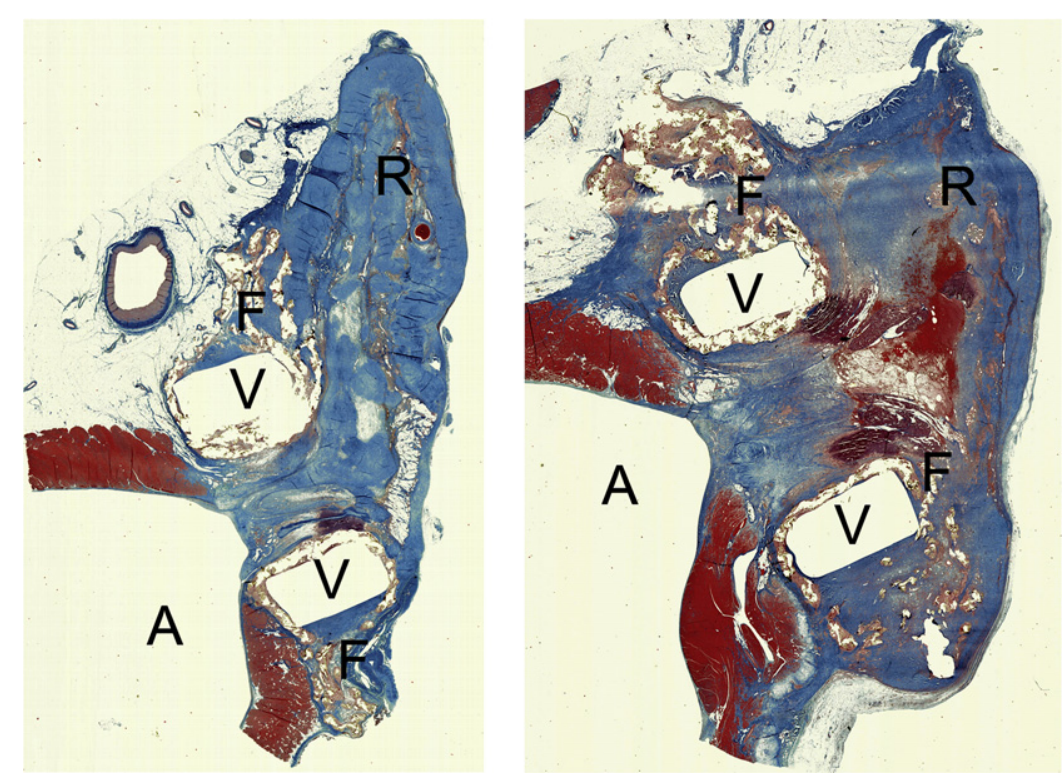

FIGURE 5. Histopathology of the left and right atrial appendages at 12 weeks after implantation of polyester fabric-covered bands. The tissue was stained with Masson's trichrome stain. $R$, Appendage remnant; $F$, band fabric; $V$, band void; $A$, atrium.

LAA in all 15 dogs (Figure 2). Additional images during dye injection at the time of death verified that the occlusion was maintained over the duration of follow-up. Fluoroscopic images obtained without dye injection showed that there was no band migration at any time after deployment (Figure 3).

\section{Gross Pathology}

A gross pathologic examination was performed in each animal after death (Figure 4). The bands and appendage remnants were encapsulated by fibrous adhesions. At 12 weeks, the adhesions were very stable and could not be moved with moderate tension. No pericarditis or other medical complications from tissue abrasion caused by the implant were observed. The atrial appendages became necrotic, and the appendage remnant distal to the occluding bands was consistently small, firm, and pale compared with the atrial tissue proximal to the bands. On the atrial endocardial surface, the occlusion site generally consisted of folded or puckered tissue, with red discoloration (endocardial congestion and hemorrhage) delineating the border between the proximal viable tissue and the distal banded tissue. All of the occlusion sites were closed at necropsy with the band in place, with no opening to the appendage from the atrium. No cracks in or other damage to the bands were observed during postexplant inspection.

\section{Histopathology}

A fibrous scar sealing each appendage remnant was intact and lined by endothelium, with no thrombotic material observed (Figure 5). Cardiac myocytes within the atrial wall at the scar boundary were often atrophied, with clusters of myocytes occasionally isolated by fibrocollagenous tissue.
Mild chronic inflammation was occasionally observed within vascular areas of the fibrous atrial scar but did not extend into atrial cardiac tissue. Connective tissue forming the scar had areas of chondroid deposition, cartilage, and bone formation. In addition, no intravascular thromboemboli were observed in tissue sections of the lung, liver, left and right kidneys, or brain.

\section{DISCUSSION}

The goal of this study was to develop and evaluate a novel band occlusion device that could be used to isolate the atrial appendage. The band and associated delivery tool that were developed could be rapidly deployed from a thoracoscopic approach but could also be deployed through a sternotomy or other surgical incision. Importantly, there were no major complications associated with deployment of the occlusive bands and no migration of the bands over time. The polyester fabric-coated bands used in the present study were associated with adhesions; however, there was no pericarditis or other medical complications from tissue abrasion caused by the implants. Furthermore, the most important finding of the present study was that band implantation resulted in $100 \%$ exclusion of the RAA and LAA from the rest of the atria. The success of exclusion was confirmed by means of both radiopaque dye injection and direct postmortem examination.

Postmortem examination showed that the atrial appendages became necrotic and the appendage remnant distal to the occluding bands was consistently small, firm, and pale compared with the atrial tissue proximal to the bands. On the atrial endocardial surface, the occlusion site generally consisted of folded or puckered tissue, with red 
discoloration delineating the border between the proximal viable tissue and the distal banded tissue. Histopathology showed a fibrous scar sealing each appendage remnant that was lined by endothelium. Although the only anticoagulant given to the animals was $81 \mathrm{mg}$ of aspirin for 1 week after implantation, no thrombotic material was observed.

The rate of complete exclusion of the LAA observed with band occlusion in the present study is higher than the rate of complete occlusion by suture or staple exclusion of the LAA in patients reported by Kanderian and coworkers. ${ }^{7} \mathrm{Al}-$ though band occlusion was not compared with suture or stapler exclusion in the present study, band occlusion might be superior because the band continues to apply circumferential force to the atrial appendage as the tissue becomes necrotic.

A limitation of the present study is that the occlusive bands were applied to normal atria in healthy canines. Patients who would be candidates for surgical atrial exclusion often have enlarged left atria ${ }^{10}$ and might require a band with a larger diameter than the bands used on the canine atria in the present study. Furthermore, the appendages of older patients with various comorbidities might be more friable and prone to tearing than those of the relatively young and healthy canines used in this study. Future studies will investigate the band occlusion method for larger and broader-based appendages more representative of the size of the LAA in patients. Indeed, a multicenter phase II clinical trial is currently underway in the United States to demonstrate successful occlusion in human subjects.

\section{CONCLUSIONS}

Permanent occlusion of the LAA is feasible via a thoracoscopic epicardial approach with this novel banding tech- nique. As the atrial tissue becomes necrotic, the silicone band continues to constrict, ensuring that the appendage remains isolated. Band occlusion of the LAA could potentially improve overall treatment outcomes in patients at high risk of atrial thrombus formation.

\section{References}

1. Blackshear JL, Odell JA. Appendage obliteration to reduce stroke in cardiac surgical patients with atrial fibrillation. Ann Thorac Surg. 1996;61:755-9.

2. Manning WJ, Silverman DI, Katz SE, Riley MF, Come PC, Doherty RM, et al. Impaired left atrial mechanical function after cardioversion: relation to the duration of atrial fibrillation. J Am Coll Cardiol. 1994;23:1535-40.

3. Bonow RO, Carabello BA, Chatterjee K, de Leon AC Jr, Faxon DP, Freed MD, et al. ACC/AHA 2006 guidelines for the management of patients with valvular heart disease: a report of the American College of Cardiology/American Heart Association Task Force on Practice Guidelines (Writing Committee to Revise the 1998 Guidelines for the Management of Patients With Valvular Heart Disease): developed in collaboration with the Society of Cardiovascular Anesthesiologists: endorsed by the Society for Cardiovascular Angiography and Interventions and the Society of Thoracic Surgeons. J Am Coll Cardiol. 2006;48:e1-148.

4. Healey JS, Crystal E, Lamy A, Teoh K, Semelhago L, Hohnloser SH, et al. Left Atrial Appendage Occlusion Study (LAAOS): results of a randomized controlled pilot study of left atrial appendage occlusion during coronary bypass surgery in patients at risk for stroke. Am Heart J. 2005;150:288-93.

5. Katz ES, Tsiamtsiouris T, Applebaum RM, Schwartzbard A, Tunick PA, Kronzon I. Surgical left atrial appendage ligation is frequently incomplete: a transesophageal echocardiographic study. J Am Coll Cardiol. 2000;36:468-71.

6. García-Fernández MA, Pérez-David E, Quiles J, Peralta J, García-Rojas I, Bermejo J, et al. Role of left atrial appendage obliteration in stroke reduction in patients with mitral valve prosthesis: a transesophageal echocardiographic study. J Am Coll Cardiol. 2003;42:1253-8.

7. Kanderian AS, Gillinov AM, Pettersson GB, Blackstone E, Klein AL. Success of surgical left atrial appendage closure: assessment by transesophageal echocardiography. J Am Coll Cardiol. 2008;52:924-9.

8. Odell JA, Blackshear JL, Davies E, Byrne WJ, Kollmorgen CF, Edwards WD, et al. Thoracoscopic obliteration of the left atrial appendage: potential for stroke reduction? Ann Thorac Surg. 1996;61:565-9.

9. Blackshear JL, Johnson WD, Odell JA, Baker VS, Howard M, Pearce L, et al. Thoracoscopic extracardiac obliteration of the left atrial appendage for stroke risk reduction in atrial fibrillation. J Am Coll Cardiol. 2003;42:1249-52.

10. Abhayaratna WP, Seward JB, Appleton CP, Douglas PS, Oh JK, Tajik AJ, et al. Left atrial size: physiologic determinants and clinical applications. J Am Coll Cardiol. 2006;47:2357-63. 